\title{
СТІЙКІСТЬ НЕТОНКИХ АНІЗОТРОПНИХ КОНІЧНИХ ОБОЛОНОК ПІД ДІЄЮ ОСЬОВОГО СТИСКУ
}

\section{STABILITY NON-THIN ANISOTROPIC CONICAL SHELLS UNDER AXIAL COMPRESSION}

Трач В.М., д.т.н., проф., ORCID ID: 0000-0001-9500-2743 Хоружий М.М., к.т.н., ORCID ID: 0000-0002-4546-9270 (Національний університет водного господарства та природокористування)

Trach V.M., Doctor of Technical Sciences, Professor, ORCID ID: 0000-00019500-2743 Khoruzhyi M.M., PhD., ORCID ID: 0000-0002-4546-9270 (National University of Water and Environmental Engineering)

Представлено підхід до розрахунку на стійкість нетонких конічних оболонок при урахуванні геометрично нелінійного докритичного напружено-деформованого стану. Представлені графіки, що ілюструють вплив кута укладання шаруватих волокнистих композитів на величини критичних значень осьового стиску.

Cone structures in the form of cones are widely used in shipbuilding, aircraft construction, instrument making, rocket technology, construction, engineering, and many other industries. The use of laminated composite materials can enhance one of their main advantages - a combination of ease with high durability. When designing thin-walled shell designs one of the main steps is to calculate the stability.

The paper presents an approach to calculations for the stability of non-thin conical anisotropic membranes made from a material having a plane of elastic symmetry based on a refined theory of the Tymoshenko-Midline type. The material of which the shell is made has one plane of elastic symmetry, which is due to the rotation of the principal directions of elasticity of the output orthotropic material.

To construct equations that help determine the critical state of the shells associated with the phenomenon of bifurcation, we use the canonical system of equations for nonlinear deformation of symmetrically loaded non-thin anisotropic shells.

The problem of static stability of a symmetrically loaded elastic anisotropic rotation shell is reduced to a system of ten ordinary homogeneous differential equations in normal form with variable coefficients and homogeneous boundary conditions.

The method of solving the boundary value problem under consideration is 
based on the numerical method of discrete orthogonalization. The numerical methodology for calculating the task is implemented as a software package for the PC.

To represent the proposed method, we consider the problem of calculating the stability of a hinged conic shell made from boron plastic. Axial compression is applied to the shell. The results of the calculations are presented in the form of graphs illustrating the dependence of the magnitude of the critical value of the axial compression on the change in the angle of the composite winding and the angle of the shell conic. The obtained critical loads are compared with numerous calculations for the stability of anisotropic shells, using a technique that relies on the Kirchhoff-Love hypothesis.

Ключові слова: стійкість, анізотропія, оболонка, конус, композит, стиск stability, anisotropy, shell, cone, composite, compression.

Вступ. Оболонкові конструкції у вигляді конусів широко застосовуються в суднобудуванні, авіабудуванні, приладобудуванні, ракетній техніці, будівництві, машинобудуванні та в багатьох інших галузях промисловості. Використання композиційних матеріалів дозволяє посилити одне 3 головних їхніх переваг - поєднання легкості з високою міцністю. Істотний інтерес 3 точки зору вимог практики розрахунку і проектування багатошарових конструкцій представляє аналіз впливу структури пакета, геометричних i механічних параметрів шарів, схем армування та інших факторів на характеристики, що визначають несну здатність тонкостінних шаруватих елементів конструкцій, яка, як відомо, найчастіше пов'язана 3 втратою стійкості.

Аналіз останніх досліджень. Аналізуючи стан досягнень в галузі механіки тонкостінних конструкцій, можна констатувати наявність великої кількості напрямків, за якими відбувається процес дослідження стійкості оболонкових конструкцій, що виготовлені з композитів [1-6]. Анізотропні оболонки виготовляються, як правило, методом намотування або викладки на оправлення окремих композитних шарів, що мають незначну товщину $[4,7$, 8]. Це дозволяє вважати, що найнижчий рівень симетрії матеріалу таких шарів складає наявність однієї площини, в якій механічні властивості симетричні. Дослідження стійкості тонких анізотропних оболонок із матеріалу що має площину пружної симетрії детально преставлено в монографії [9]. В роботі, на основі нелінійної теорії, що використовує гіпотези Кірхгофа-Лява, викладені методика розрахунку та результати дослідження стійкості тонких анізотропних оболонок обертання нульової, додатної та від'ємної гауссових кривин, що виготовлені 3 композитних матеріалів з однією площиною пружної симетрії.

Проте, залишається ще досить значна кількість нез'ясованих питань щодо стійкості нетонких анізотропних оболонок. Відомо, що для розрахунку таких 
конструкцій необхідно використовувати уточнені теорії [2, 10-11], найбільшого широкого використання серед яких набула уточнена теорія, що спирається на гіпотези С. П. Тимошенка.

Постановка мети і задач досліджень. У роботі представлено підхід до розрахунків на стійкість нетонких анізотропних оболонок, що виготовлені із матеріалу, який має одну площину пружної симетрії, при використанні уточненої теорії типу Тимошенко.

Для побудови рівнянь, за допомогою яких визначається критичний стан оболонок, пов'язаний 3 явищем біфуркації, використовується канонічна система рівнянь нелінійного деформування симетрично завантажених нетонких анізотропних оболонок.

Представимо переміщення $u, v, w$ у відповідності до гіпотез Тимошенко у вигляді лінійних розкладів по координаті $z$ :

$$
\begin{gathered}
u=u_{0}+z \theta_{1}, v=v_{0}+z \theta_{2}, w=w, \\
u_{\alpha}=u_{0 \alpha}+z \theta_{\alpha}, u_{3}=w, u_{1}=u+z \theta_{1}, v_{2}=v+z \theta_{2}, w_{1}=w,
\end{gathered}
$$

де $u, v, w$ - переміщення серединної поверхні оболонки, $z$ - координата по якій змінюється товщина оболонки, $\theta_{\alpha},(\alpha=1,2)$ - кути повороту відносно нормальних до поверхні осей координат.

Співвідношення закону Гука для анізотропного матеріалу з однією площиною пружної симетрії згідно [1] мають вид:

$$
\begin{gathered}
\sigma_{11}=a_{11} \varepsilon_{11}+a_{12} \varepsilon_{22}+a_{13} \varepsilon_{33}+a_{16} \varepsilon_{12}, \\
\sigma_{12}=a_{16} \varepsilon_{11}+a_{26} \varepsilon_{22}+a_{36} \varepsilon_{33}+a_{66} \varepsilon_{12}, \\
\sigma_{22}=a_{12} \varepsilon_{11}+a_{22} \varepsilon_{22}+a_{23} \varepsilon_{33}+a_{26} \varepsilon_{12}, \\
\sigma_{13}=a_{45} \varepsilon_{23}+a_{55} \varepsilon_{13}, \sigma_{23}=a_{44} \varepsilon_{23}+a_{45} \varepsilon_{13}, \\
\sigma_{33}=a_{13} \varepsilon_{11}+a_{23} \varepsilon_{22}+a_{33} \varepsilon_{33}+a_{36} \varepsilon_{12},
\end{gathered}
$$

де $\sigma_{i j}$ - нормальні і зсувні напруження, $\varepsilon_{i j}$ - деформації розтягу і зсуву ( $i, j=1,2,3), a_{i j}$ - пружні сталі $(i=1,2,3 ; j=\overline{1,6})$.

Для нелінійних деформацій з точністю до квадратичних членів в [2] отримано:

$$
\begin{gathered}
\varepsilon_{11}=\bar{\varepsilon}_{11}+z k_{11}+z^{2} v_{11}, \varepsilon_{22}=\bar{\varepsilon}_{22}+z k_{22}+z^{2} v_{22}, \\
\varepsilon_{12}=\bar{\varepsilon}_{12}+z k_{12}+z^{2} v_{12} .
\end{gathered}
$$

В [9] представлено підхід до виведення системи канонічних рівнянь, яка описує осесиметричний напружено-деформований стан тонких анізотропних оболонок. За його сприяння в роботі отримані рівняння нелінійного деформування симетрично завантажених нетонких анізотропних оболонок: 
$\frac{1}{A_{1}} \frac{\partial u}{\partial \alpha_{1}}=b_{11} T_{11}+b_{12} T_{12}+b_{13} M_{11}+b_{14} M_{12}+b_{11}^{\prime} \frac{u}{A_{1} A_{2}} \frac{\partial A_{2}}{\partial \alpha_{1}}-$ $-b_{11}^{\prime} \frac{w}{R_{2}}-b_{11}^{\prime \prime} \frac{1}{A_{1} A_{2}} \frac{\partial A_{2}}{\partial \alpha_{1}} \theta_{1}-\frac{v}{A_{1} A_{2}} \frac{\partial A_{2}}{\partial \alpha_{1}}+\frac{w}{R_{2}}+\frac{1}{2} \theta_{2}^{2} ;$

$\frac{1}{A_{1}} \frac{\partial v}{\partial \alpha_{1}}=b_{21} T_{11}+b_{22} T_{12}+b_{23} M_{11}+b_{24} M_{12}+b_{21}^{\prime} \frac{u}{A_{1} A_{2}} \frac{\partial A_{2}}{\partial \alpha_{1}}-$ $-b_{21}^{\prime} \frac{w}{R_{2}}-b_{21}^{\prime \prime} \frac{1}{A_{1} A_{2}} \frac{\partial A_{2}}{\partial \alpha_{1}} \theta_{1}+\frac{v}{A_{1} A_{2}} \frac{\partial A_{2}}{\partial \alpha_{1}}+\frac{1}{2} \theta_{2}^{2} ;$

$\frac{1}{A_{1}} \frac{\partial w}{\partial \alpha_{1}}=b_{55} T_{13}+b_{55}^{\prime} T_{23}-\frac{u}{R_{1}}-\theta_{1}$;

$\frac{1}{A_{1}} \frac{\partial \theta_{1}}{\partial \alpha_{1}}=b_{31} T_{11}+b_{32} T_{12}+b_{33} M_{11}+b_{34} M_{12}+b_{31}^{\prime} \frac{u}{A_{1} A_{2}} \frac{\partial A_{2}}{\partial \alpha_{1}}-$ $-b_{31}^{\prime} \frac{w}{R_{2}}-b_{31}^{\prime \prime} \frac{1}{A_{1} A_{2}} \frac{\partial A_{2}}{\partial \alpha_{1}} \theta_{1}+\frac{1}{2} \theta_{2}^{2} ;$

$\frac{1}{A_{1}} \frac{\partial \theta_{2}}{\partial \alpha_{1}}=b_{41} T_{11}+b_{42} T_{12}+b_{43} M_{11}+b_{44} M_{12}+b_{41}^{\prime} \frac{u}{A_{1} A_{2}} \frac{\partial A_{2}}{\partial \alpha_{1}}-$ $-b_{41}^{\prime} \frac{w}{R_{2}}-b_{41}^{\prime \prime} \frac{1}{A_{1} A_{2}} \frac{\partial A_{2}}{\partial \alpha_{1}} \theta_{1}+\frac{1}{A_{1} A_{2}} \frac{\partial A_{2}}{\partial \alpha_{1}} \theta_{2}+\frac{1}{2} \theta_{2}^{2} ;$

$\frac{1}{A_{1}} \frac{\partial T_{11}}{\partial \alpha_{1}}=-\frac{1}{A_{2}} \frac{\partial T_{12}^{*}}{\partial \alpha_{2}}-\frac{1}{A_{1} A_{2}} \frac{\partial A_{2}}{\partial \alpha_{1}} T_{22}-\frac{1}{R_{1}} T_{13}-q_{1} ;$

$\frac{1}{A_{1}} \frac{\partial T_{12}^{*}}{\partial \alpha_{1}}=\frac{1}{A_{1} A_{2}} \frac{\partial A_{2}}{\partial \alpha_{1}} T_{12}^{*}-\frac{1}{R_{2}} T_{23} ;$

$\frac{1}{A_{1}} \frac{\partial T_{13}}{\partial \alpha_{1}}=-\frac{1}{R_{1}} T_{11}-\frac{1}{R_{2}} T_{22}-q_{3}$;

$\frac{1}{A_{1}} \frac{\partial M_{11}}{\partial \alpha_{1}}=\frac{1}{A_{2}} \frac{\partial M_{12}^{*}}{\partial \alpha_{2}}+\frac{1}{A_{1} A_{2}} \frac{\partial A_{2}}{\partial \alpha_{1}} M_{22}+T_{13}+T_{11} \theta_{1} ;$

$\frac{1}{A_{1}} \frac{\partial M_{12}^{*}}{\partial \alpha_{1}}=\frac{1}{A_{1} A_{2}} \frac{\partial A_{2}}{\partial \alpha_{1}} M_{12}^{*}-\frac{1}{A_{1} A_{2}} \frac{\partial A_{1}}{\partial \alpha_{2}} M_{11}-T_{23}+T_{22} \theta_{2}$. 
Система нелінійних рівнянь (4) може бути використана для побудови рівнянь, за допомогою яких визначається критичний стан оболонки, пов'язаний з явищем біфуркації. Після введення позначень:

$$
\begin{gathered}
y_{1}=u, \quad y_{2}=v, \quad y_{3}=w, \quad y_{4}=\theta_{1}, \quad y_{5}=\theta_{2}, \\
y_{6}=T_{11}, \quad y_{7}=T_{12}^{*}, \quad y_{8}=T_{13}, \quad y_{9}=M_{11}, \quad y_{10}=M_{12}^{*} .
\end{gathered}
$$

Отримаємо систему рівнянь (4) в загальному вигляді:

$$
\frac{1}{A_{1}} \frac{\partial y_{i}}{\partial \alpha_{1}}=L_{i}(y)+q_{i}
$$

де $y$ - вектор компонентами якого $є$ функції $y_{i}, q_{i}$ - компоненти навантаження, $L_{i}$ - нелінійні диференціальні оператори, $i=1, \ldots, 10$.

На основній траєкторії деформування рівняння (6) мають вид:

$$
\frac{1}{A_{1}} \frac{\partial y_{i, o}}{\partial \alpha_{1}}=L_{i}\left(y_{o}\right)+q_{i}
$$

На суміжній траєкторії їх потрібно записати наступним чином:

$$
\frac{1}{A_{1}} \frac{\partial\left(y_{i, o}+y_{i}\right)}{\partial \alpha_{1}}=L_{i}\left(y_{o}+y\right)+q_{i} .
$$

У відповідності до критерію Ейлера, $y_{i}$ - це нескінченно малі збурення основного стану. Тому, користуючись поняттям похідної Фреше, можемо обмежитись в рядах Тейлора тільки двома членами:

$$
L_{i}\left(y_{O}+y\right)=L_{i}\left(y_{o}\right)+L_{i},{ }_{j}\left(y_{o}\right) y,
$$

де $L_{i}, j-$ похідні Фреше від операторів $L_{i}$ за аргументом $y_{j}(j=1, \ldots, 10)$.

Рівняння (8) набирають виду:

$$
\frac{1}{A_{1}} \frac{\partial y_{i, o}}{\partial \alpha_{1}}+\frac{1}{A_{1}} \frac{\partial y_{i}}{\partial \alpha_{1}}=L_{i}\left(y_{o}\right)+L_{i, j}\left(y_{o}\right) y+q_{i}
$$

Враховуючи те, що навантаження $q_{i}$ не змінюється, а функції з індексом «о» задовольняють рівнянням (7), із (10) отримаємо лінеаризовані рівняння відносно приростів функції в точці біфуркації:

$$
\frac{1}{A_{1}} \frac{\partial y_{i}}{\partial \alpha_{1}}=L_{i, j}\left(y_{o}\right) y
$$

В розгорнутій формі система диференціальних рівнянь (11) має вигляд:

$$
\frac{1}{A_{1}} \frac{\partial y_{1}}{\partial \alpha_{1}}=-\frac{1}{A_{2}} \frac{\partial T_{21}^{*}}{\partial \alpha_{2}}-a_{1}\left(y_{2}+T_{21}^{*}\right)-a_{2}\left(y_{1}-T_{21}^{*}\right)+\frac{1}{R_{1}} y_{3}
$$




$$
\begin{aligned}
& \frac{1}{A_{1}} \frac{\partial y_{2}}{\partial \alpha_{1}}=-\frac{1}{A_{2}} \frac{\partial T_{22}^{*}}{\partial \alpha_{2}}-a_{2}\left(y_{2}+T_{21}^{*}\right)+a_{1}\left(y_{1}-T_{22}^{*}\right)+\frac{1}{R_{2}} T_{23}^{*} \\
& \frac{1}{A_{1}} \frac{\partial y_{3}}{\partial \alpha_{1}}=-\frac{1}{A_{2}} \frac{\partial T_{23}^{*}}{\partial \alpha_{2}}-a_{2} y_{3}-a_{1} T_{23}^{*}-\frac{1}{R_{1}} y_{1}-\frac{1}{R_{2}} T_{22}^{*} \\
& \frac{1}{A_{1}} \frac{\partial y_{4}}{\partial \alpha_{1}}=-\frac{1}{A_{2}} \frac{\partial M_{12}}{\partial \alpha_{2}}-2 a_{1} M_{12}-a_{2}\left(y_{4}-M_{22}\right) T_{13} \\
& \frac{1}{A_{1}} \frac{\partial y_{5}}{\partial \alpha_{1}}=-a_{1} y_{12}+\frac{1}{R_{1}} y_{7}-\varepsilon_{1} \varepsilon_{1,0}-\theta_{1} \theta_{1}^{0}+A_{11} T_{11}+A_{12} T_{12}+A_{13} y_{4}+ \\
&+d_{11} \varepsilon_{22}+d_{12} \kappa_{22}+d_{13} \kappa_{12} \\
& \frac{1}{A_{1}} \frac{\partial y_{6}}{\partial \alpha_{1}}= a_{1} y_{5}-\theta_{1,0} \theta_{2}-\theta_{1} \theta_{2,0}+A_{21} T_{11}+A_{22} T_{12}+A_{23} y_{4}+ \\
&+d_{21} \varepsilon_{22}+d_{22} \kappa_{22}+d_{23} \kappa_{12} ; \\
& \frac{1}{A_{1}} \frac{\partial y_{7}}{\partial \alpha_{1}}=-\frac{1}{R_{1}} y_{5}-y_{8} ; \\
& \frac{1}{A_{1}} \frac{\partial y_{8}}{\partial \alpha_{1}}= a_{1} \theta_{2}+A_{31} T_{11}+A_{32} T_{12}+A_{33} y_{4}+d_{31} \varepsilon_{22}+d_{32} \kappa_{22}+d_{33} \kappa_{12} \\
& \frac{1}{A_{1}} \frac{\partial y_{9}}{\partial \alpha_{1}}= T_{23}^{*}+d_{31} \varepsilon_{22}+d_{32} \kappa_{22}+d_{33} \kappa_{12} \\
& \frac{1}{A_{1}} \frac{\partial y_{10}}{\partial \alpha_{1}}=M_{12}^{*}+A_{32} T_{12}^{*}+d_{33} \kappa_{12}
\end{aligned}
$$

де введені такі позначення:

$$
\begin{gathered}
y_{1}=u, y_{2}=v, y_{3}=w, y_{4}=\theta_{1}, y_{5}=\theta_{2} \\
y_{6}=T_{11}\left(1+\varepsilon_{1,0}\right)+T_{11,0} \varepsilon_{1}, y_{7}=T_{12}\left(1+\varepsilon_{2,0}\right)+T_{12,0} \varepsilon_{2}-\frac{2}{R_{2}} M_{12} \\
y_{8}=T_{13}+T_{11,0} \theta_{1}+T_{11,0} \theta_{1,0}+T_{12} \theta_{2}+T_{12} \theta_{2,0}, y_{9}=M_{11}, y_{10}=M_{12}^{*} \\
T_{21}^{*}=T_{12}\left(1+\varepsilon_{1,0}\right), T_{22}^{*}=T_{22}\left(1+\varepsilon_{2,0}\right)+T_{22,0} \varepsilon_{2} \\
T_{23}^{*}=\frac{1}{A_{2}} \frac{\partial M_{22}}{\partial \alpha_{2}}-a_{1}\left(y_{4}-M_{22}\right)+2 a_{2} M_{12}+ \\
+T_{11,0} \theta_{1}+T_{11} \theta_{1,0}+T_{12,0} \theta_{2}+T_{12} \theta_{2,0} .
\end{gathered}
$$


Система (12) є однорідною системою диференціальних рівнянь другого порядку, що описує стійкість анізотропних оболонок.

Методика досліджень. Розглядувані циліндричні оболонки замкнуті в коловому напрямі, тому розв'язувальні функції періодичні за коловою координатою $\alpha_{2}$ або $\varphi$. Представимо шукані функції у вигляді рядів Фур'є в комплексній формі:

$$
y_{j}=\sum_{n=-\infty}^{n=\infty} y_{j, n} e^{i n \varphi}, \quad \varphi=\alpha_{2}, \quad 0 \leq \alpha_{2} \leq 2 \pi .
$$

де $y_{j, n}$ - комплексні функції, $j=1, \ldots, 10, n$ - число хвиль в коловому напрямі.

Після підстановки (14) в систему рівнянь стійкості (12) і після деяких перетворень, отримаємо систему звичайних диференціальних рівнянь, яка для кожного додатного значення $n$ має вигляд:

$$
\begin{aligned}
& \frac{1}{A_{1}} \frac{d y_{1, n}}{d \alpha_{1}}=-i n_{a}\left(T_{12, n}\right)+\psi_{2}\left(T_{22, n}-T_{11, n}\right)+\frac{1}{R_{1}}\left(y_{3, n}-i n_{a} M_{12, n}\right) ; \\
& \frac{1}{A_{1}} \frac{d y_{2, n}}{d \alpha_{1}}=-i n_{a}\left(\left(T_{22, n}\right)-\psi_{2}\left(2 T_{12, n}\right)+\left(\frac{3}{R_{2}}-\frac{1}{R_{1}}\right) \psi_{2} M_{12, n}+\right. \\
& \frac{1}{R_{2}}\left(-T_{12}^{0} y_{8, n}+T_{22}^{0} \theta_{2, n}-T_{12, n} y_{8}^{0}+T_{22, n} \theta_{2}^{0}+i n_{a} M_{12, n}\right) ; \\
& \frac{1}{A_{1}} \frac{d y_{3, n}}{d \alpha_{1}}=-i n_{a}\left(-T_{12}^{0} y_{8, n}+T_{22}^{0} \theta_{2, n}-T_{12, n} y_{8}^{0}+T_{22, n} \theta_{2}^{0}+2 \psi_{2} M_{12, n}\right)- \\
& \quad-\psi_{2} y_{3, n}-\frac{1}{R_{1}} y_{1, n}-\frac{1}{R_{2}}\left(T_{22, n}\right) ; \\
& \frac{1}{A_{1}} \frac{d y_{4, n}}{d \alpha_{1}}=-i n_{a} M_{12, n}-\psi_{2}\left(y_{4, n}-M_{22, n}\right)+y_{3, n}+T_{11}^{0} y_{8, n}+
\end{aligned}
$$

$$
+T_{11, n} y_{8}^{0}-T_{12}^{0} \theta_{2, n}-S_{, n} \theta_{2}^{0}
$$$$
\frac{1}{A_{1}} \frac{d y_{5, n}}{d \alpha_{1}}=\frac{1}{R_{1}} y_{7, n}-y_{8, n} y_{8}^{0}+A_{11} T_{11, n}+A_{12} T_{12, n}+A_{13} y_{4, n}-
$$$$
-d_{11} \varepsilon_{22, n}-d_{12} \chi_{22, n}-d_{13} \chi_{12, n}
$$ 


$$
\begin{aligned}
\frac{1}{A_{1}} \frac{d y_{6, n}}{d \partial \alpha_{1}}= & -i n_{a} y_{5, n}+\psi_{2} y_{6, n}+y_{8, n} \theta_{2}^{0}+y_{8}^{0} \theta_{2, n}+A_{12} T_{11, n}+A_{22} S_{, n}+ \\
& +A_{23} y_{4, n}-d_{21} \varepsilon_{22, n}-d_{22} \chi_{22, n}-d_{23} \chi_{12, n} \\
\frac{1}{A_{1}} \frac{d y_{7, n}}{d \alpha_{1}}= & -\frac{1}{R_{1}} y_{5, n}-y_{8, n} ; \\
\frac{1}{A_{1}} \frac{d y_{8, n}}{d \alpha_{1}}= & -\frac{1}{R_{1}} \varepsilon_{1, n}+A_{13} T_{11, n}+A_{23} S_{, n}+A_{33} y_{4, n}-d_{31} \varepsilon_{22, n}- \\
& -d_{32} k_{22, n}-d_{33} k_{12, n} \\
\frac{1}{A_{1}} \frac{\partial y_{9, n}}{\partial \alpha_{1}}= & T_{23, n}^{*}+d_{31} \varepsilon_{22, n}+d_{32} \kappa_{22, n}+d_{33} \kappa_{12, n} \\
\frac{1}{A_{1}} \frac{\partial y_{10, n}}{\partial \alpha_{1}}= & M_{12, n}^{*}+A_{32} T_{12, n}^{*}+d_{33} \kappa_{12, n}
\end{aligned}
$$

де $n_{a}=n / A_{2}$.

Таким чином, задача статичної стійкості симетрично завантаженої пружної анізотропної оболонки обертання, замкнутої в коловому напрямі, зведена до системи з десяти звичайних однорідних диференціальних рівнянь у нормальній формі (15) із змінними коефіцієнтами і однорідними граничними умовами:

$$
\begin{array}{lll}
\text { - на контурі } \alpha_{1}=\alpha_{0}, & B_{o} y_{n}=0 ; \\
\text { - на контурі } \alpha_{1}=\alpha_{l}, & B_{n} y_{n}=0 .
\end{array}
$$

Мінімальне власне значення однорідної крайової задачі (15), (16) характеризує момент переходу від симетричного основного рівноважного стану до несиметричного, якому властиві відповідне число хвиль втрати стійкості в коловому напрямку. Цей стан рівноваги повністю характеризується такими функціями: $y_{1, n}, \ldots y_{10, n}, T_{11, n}, T_{22, n}, T_{12, n}$, $M_{22, n}, M_{12, n}, \quad \varepsilon_{1, n}, \varepsilon_{2, n}, \varepsilon_{22, n}, \theta_{1, n}, \theta_{2, n}, \mathrm{k}_{22, n}, \mathrm{k}_{12, n}$, а також докритичними параметрами $T_{11}^{0}, T_{22}^{0}, \mathrm{~T}_{12}^{0}, \theta_{1}^{0}, \theta_{2}^{0}$.

Мінімальне власне число знаходиться при послідовному збільшенні навантаження, коли визначник матриці “правих" граничних умов дорівнює нулю. При розв'язку системи диференціальних рівнянь 3 комплексними коефіцієнтами визначник також $\epsilon$ комплексним. Щоб існував розв'язок системи однорідних алгебраїчних рівнянь 3 комплексними коефіцієнтами 
необхідно, щоб були одночасно рівними нулю дійсна та уявна частини визначника.

Методика розв’язку, розглядуваної крайової задачі, базується на чисельному методі дискретної ортогоналізації

Результати досліджень. Для представлення запропонованої методики розглянута задача про стійкість одношарової конічної оболонки (рис. 1). При проведенні розрахунків будемо розглядати оболонки, що виготовлені iз боропластика. У власних осях цей матеріал ортотропний. Відносно координатних осей на серединній поверхні оболонки осі ортотропії можуть бути повернуті на довільний кут $\psi$. Тоді при $\psi \neq 0^{\circ}, 90^{\circ}$ він втрачає властивості ортотропії та веде себе при деформуванні як матеріал з однією площиною пружної симетрії.

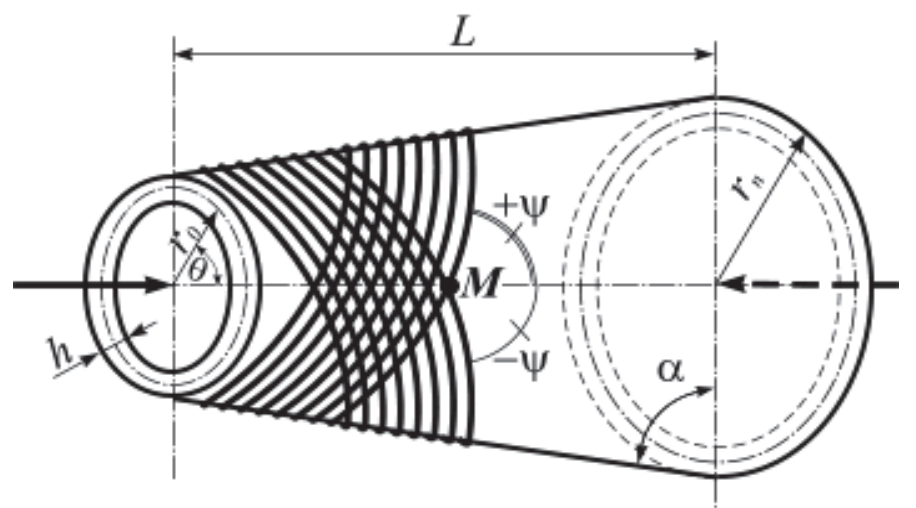

Рис.1. Розрахункова схема конічної оболонки

Дослідження стійкості проводились для оболонок із відношенням товщини до iii радіуса $h / R=0,1$. Геометрія таких оболонок описана наступними співвідношеннями:

$$
R_{0}=r_{0} / \sin \alpha ; R_{n}=r_{n} / \sin \alpha ; L=\left(r_{n}-r_{0}\right) / \cos \alpha,
$$

де $\alpha$ - кут нахилу твірної оболонки до основи більшого радіуса, $r_{0}$ - радіус меншої основи конуса, $r_{n}$ - радіус більшої основи конуса. Для досліджуваних оболонок прийнято, що $r_{0}=1,0 \mathcal{M}$. Висота конічних оболонок, для всіх розглянутих значень кута $\alpha$, була сталою та рівною $L=1,0 \mathrm{M}$. Проведено розрахунки на стійкість анізотропних оболонок для кутів $\alpha$, рівних $80^{\circ}, 70^{\circ}$ та $60^{\circ}$.

Закріплення торців оболонки наступне: один торець закріплений шарнірно-рухомо $v=w=M_{11}=M_{12}=0, T_{11}=-T_{11}^{0}$ а інший шарнірнонерухомо $\left(u=v=w=M_{11}=M_{12}=0\right)$.

На рис. 2 представлені графіки, що ілюструють вплив кута намотування волокнистого композиту $\psi_{i}$ на величини критичних значень осьових зусиль 
$T_{11}^{c r}$. Крива 1 - це критичні значення, здобуті за розробленою методикою Графічна залежність 2 являє собою критичні осьові стискаючі навантаження $T_{11}^{c r}$, знайдені за методикою розрахунку на стійкість анізотропних оболонок, що представлена в [9] і базується на гіпотезах Кірхгофа-Лява.

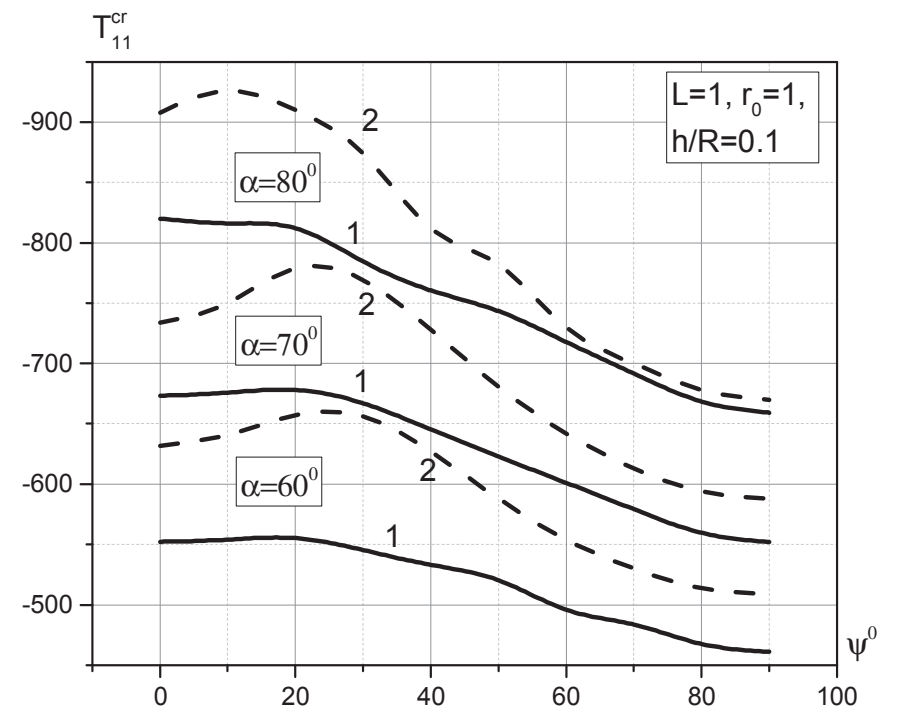

Рис.2. Критичні значення осьових стискаючих зусиль

Висновки. Аналіз графіків, представлених на рис. 2 дозволяє зробити такі висновки. Основний пропонований підхід розрахунку на стійкість конічних анізотропних оболонок, що знаходяться під дією осьового стискаючого навантаження, призводять до отримання найменших величин критичних навантажень порівняно із методикою, що базується на гіпотезах КірхгофаЛява. Із зменшенням кута конусності $\alpha$ зменшується і величина критичного навантаження. Для оболонок, товщина яких становить $h=0,1 R$, максимальна розбіжність між значеннями критичного навантаження, порахованого за двома підходами складає при дії осьового стиску 13,5\% для конічної оболонки з $\alpha=80^{\circ}, 15,3 \%$ - з $\alpha=70^{\circ}$ та $20,3 \%-3 \alpha=60^{\circ}$.

1. Амбарцумян С.А. Общая теория анизотропных оболочек / С.А. Амбарцумян. М.: Наука, 1974. - 448 с.

2. Ванин Г.А. Устойчивость оболочек из композиционных материалов с несовершенствами / Г.А. Ванин, М.П. Семенюк. - Київ: Наукова думка, 1987. - 200 с.

3. Вольмир А.С. Устойчивость деформируемых систем / А.С. Вольмир. - М.: Наука, 1967. $-984 \mathrm{c}$.

4. Гузь А.Н. Основы трехмерной теории устойчивости деформируемых тел / А.Н. Гузь. - К.: Вища школа, 1986. -512 с.

5. Кармишин А.В. Статика и динамика тонкостенных оболочечных конструкций / А.В. Кармишин, В.А. Ласковец, В.И. Мяченков, А.Н. Фролов. - М.: Машиностроение, 1975. $376 \mathrm{c}$.

6. Рикардс Р.Б. Устойчивость оболочек из композитных материалов / Р.Б. Рикардс, Г.А. Тетерс. - Рига: Зинатне, 1974. - 310 с. 
7. Королев В.И. Слоистые анизотропные пластинки и оболочки из армированных пластмасс / В.И. Королев. - М.: Машиностроение, 1965. - 272 с.

8. Микишева В.М. Оптимальная намотка оболочек из стеклопластика работающих на устойчивость под внешним давлением или осевым сжатием / В.М. Микишева // Механика полимеров. - Рига, Знание, 1968. - №5. - С. 864-875.

9. Баженов В.А. Нелінійне деформування, стійкість і закритична поведінка анізотропних оболонок / В.А. Баженов, М.П. Семенюк, В.М. Трач. - К.: Каравела, 2010. - 352 с.

10. Семенюк Н.П. Об учете кубической нелинейности в уравнениях теории анизотропных оболочек / Н.П. Семенюк, Н.Б. Жукова, В.М. Трач, М.М. Хоружий // Прикладная механика. - 2012. - Т. 48, № 6. - С. 124-134.

11. Trach W. Stateczność osiowo ściskanych cylindrycznych, anizotropowych powłok o średniej grubości / W. Trach, M. Chorużyj // Acta Scientiarum Polonorum - Architectura (Budownictwo). - 2015. - no 14 (3). - pp. 21-34.

1. Ambartsumyan S.A. Obschaya teoriya anizotropnyh obolochek [General theory of anisotropic shells]. Moscow: Nauka, 1978.

2. Vanin G.A., Semenyuk M.P. Ustoychivost obolochek iz kompozitsionnyh materialov s nesovershenstvami [Stability of shells from composite materials with imperfections]. Kyiv: Naukova dumka, 1987.

3. Volmir A.S. Ustoychivost deformiruemyh sistem [Stability of deformable systems]. Moscow: Nauka, 1967.

4. Guz A.N. Osnovy trehmernoy teorii ustoychivosti deformiruemyh tel [Fundamentals of the three-dimensional theory of stability of deformable bodies]. Kyiv: Vischa shkola, 1986.

5. Karmishin A.V., Laskovets V.A., Myachenkov V.I., Frolov A.N. Statika i dinamika tonkostennyh obolochechnyh konstruktsiy [Statics and dynamics of thin-walled shell structures]. Moscow: Mashinostroenie, 1975.

6. Rikards R.B., Teters G.A. Ustoychivost obolochek iz kompozitnyh materialov [Stability of shells of composite materials]. Riga: Zinatne, 1974.

7. Korolev V.I. Sloistye anizotropnye plastinki i obolochki iz armirovannyh plastmass [Layered anisotropic plates and shells of reinforced plastics]. Moscow: Mashinostroenie, 1965.

8. Mikisheva V.M. Optimalnaya namotka obolochek iz stekloplastika rabotayuschih na ustoychivost pod vneshnim davleniem ili osevym szhatiem [Optimum winding of fiberglass shells working on stability under external pressure or axial compression], Mehanika polimerov. Riga, Znanie, №5, pp. 864-875, 1968.

9. Bazhenov V.A., Semeniuk M.P., Trach V.M. Neliniine deformuvannia, stiikist i zakrytychna povedinka anizotropnykh obolonok [Nonlinear deformation, stability and supercritical behavior of anisotropic shells]. Kyiv: Karavela, 2010.

10. Semenyuk N.P., Zhukova N.B., Trach V.M., Horuzhiy M.M. Ob uchete kubicheskoy nelineynosti $\mathrm{v}$ uravneniyah teorii anizotropnyh obolochek [On the account of cubic nonlinearity in the equations of the theory of anisotropic shells]. Prikladnaya mehanika, Vol. 48, № 6, pp. 124-134, 2012.

11. Trach W., Chorużyj M. Stateczność osiowo ściskanych cylindrycznych, anizotropowych powłok o średniej grubości. Acta Scientiarum Polonorum - Architectura (Budownictwo), no 14 (3), pp. 21-34, 2015. 